\title{
Platelet-to-Lymphocyte Ratio Predicts the Presence of Diabetic Neurogenic Bladder
}

\author{
Yiying Liu' ${ }^{\prime}, *$ \\ Xin Wang ${ }^{2, *}$ \\ Linying Wang' \\ Wenwen Chen ${ }^{3}$ \\ Wenyue Liu' \\ Tingting $\mathrm{Ye}^{\mathrm{I}}$ \\ Jing Hong $\mathbb{D}^{\prime}$ \\ Hong Zhu (D) \\ Feixia Shen' \\ 'Department of Endocrinology and \\ Metabolism, The First Affiliated Hospital \\ of Wenzhou Medical University, \\ Wenzhou, People's Republic of China; \\ ${ }^{2}$ Department of Urology, Taizhou \\ Hospital of Zhejiang Province Affiliated \\ to Wenzhou Medical University, Taizhou, \\ People's Republic of China; ${ }^{3}$ Department \\ of Senile Diseases, The First Affiliated \\ Hospital of Wenzhou Medical University, \\ Wenzhou, People's Republic of China
}

*These authors contributed equally to this work
Correspondence: Feixia Shen

Department of Endocrinology and

Metabolism, The First Affiliated Hospital

of Wenzhou Medical University,

Wenzhou, People's Republic of China

Email shenfeixia@wmu.edu.cn
Purpose: Diabetic neurogenic bladder (DNB) has been widely recognized in recent years. It is common in patients with long-term diabetes and may also lead to many severe complications. Although there has been widespread evidence that inflammation is involved in the development of some diabetic complications, there is little evidence that this can also occur in the bladder. In recent years, platelet-to-lymphocyte ratio (PLR) and neutrophil-tolymphocyte ratio (NLR) have been viewed as potential novel markers of inflammatory responses. This study was designed to evaluate the relationship between the presence of DNB and the PLR and NLR.

Patients and Methods: A total of 371 cases of T2DM patients were included in this retrospective study. Patients were divided into two groups, with 115 diabetic subjects diagnosed with diabetic neurogenic bladder and 256 control subjects without DNB. The independent predictors of DNB were analyzed using logistic regression.

Results: Compared with patients without DNB, the mean PLR and NLR were significantly higher in those with DNB $(\mathrm{p}<0.001)$. Based on the logistic regression, PLR was found to be an independent risk factor for DNB (odds ratio [OR]: $1.408,95 \%$ confidence interval [CI]: 1.248-1.617). From the receiver operating characteristic (ROC) curve, using PLR as indicative of DNB was expected to be 101.1949 , and it generated a sensitivity and specificity value of $89.6 \%$ and $23.4 \%$, respectively. The area under the curve (AUC) was also found to be 0.899 (95\% CI: $0.865-0.932$ ).

Conclusion: In our study, PLR and NLR were significantly higher for patients with DNB. The PLR was found to be a risk factor in the presence of DNB after correcting for possible confounding factors. Considering the severe complications associated with DNB, patients with elevated PLR should be seriously cared for in clinics.

Keywords: diabetic autonomic neuropathy, neurogenic bladder, oxidative stress, inflammatory response

\section{Introduction}

Diabetes mellitus (DM) is a widespread epidemic disease. According to estimates from the American Diabetes Association (ADA), more than 23 million Americans over the age of 20 have diabetes, with almost $95 \%$ of these being of type 2 (T2DM). ${ }^{1}$ Among some of the chronic complications of diabetes, there is diabetic neurogenic bladder (DNB), one type of diabetic autonomic neuropathy, which, in recent years, has been widely recognized and treated as a result of a greater understanding of the disease. Currently, based on the literature, it is estimated that morbidity in diabetic patients suffering from DNB is about $40 \%-80 \%$, while the incidence rate is still $25 \%$ despite controlling blood glucose levels. ${ }^{2}$ Further 
studies have shown that the incidence of DNB in patients with diabetes lasting more than 10 years is $25 \%$, and that for those over 15 years, can be as high as $50 \%{ }^{3}$ DNB is mainly characterized by a stable increase in post-void residual urine (PVR) and bladder volume, the weakening of bladder sensation and a decrease in bladder contractility. ${ }^{4}$ It can also further lead to urinary tract infections, uroschesis and hydronephrosis, as well as other conditions, which can seriously affect patients' quality of life. Based on the above, it is clear that improving the management of patients in an attempt to reduce patient mortality is important. At the same time, exploring effective biomarkers that can predict the presence of DNB can also be of great value.

It is well known that the pathophysiological mechanisms of T2DM are multifactorial. However, the association between chronic low-grade inflammation and DM has been the main focus of many epidemiological studies. ${ }^{5}$ However, since leukocyte (WBC) counts and its subtypes are typical inflammatory markers, ${ }^{6}$ prospective studies have attempted to use leukocyte counts, proinflammatory cytokines, chemokines, and other indirect inflammation markers (such as fibrinogen) as predictive markers of $\mathrm{T}^{2} \mathrm{DM} .^{7}$ As a result, the link between high leukocyte counts and insulin resistance has already been shown in patients with T2DM. ${ }^{8}$ Platelet-to-lymphocyte ratio (PLR) and neutrophil-to-lymphocyte ratio (NLR) are also novel biomarkers of systemic inflammation, which occur in various conditions, including cancers and cardiovascular diseases. ${ }^{9-11}$ To date, several studies have shown a relationship between diabetic microvascular complications and PLR and NLR values, ${ }^{12-14}$ with Wang and Kuo having recently found increased mast cells in the urothelium and suburothelial layers of diabetic people, ${ }^{15}$ thereby indicating a link between inflammation and bladder lesions. In this study, the aim was to investigate, for the first time, the relationship between DNB and the PLR and NLR values.

\section{Patients and Methods}

\section{Study Population}

In total, 371 patients were recruited for the study. From October 2005 to October 2020, 115 patients with type 2 diabetic neurogenic bladder conditions were selected from the Department of Endocrinology and Metabolism of The First Affiliated Hospital of Wenzhou Medical University (as DNB group). The inclusion criteria were:
1. Patients should have been diagnosed with type 2 diabetes according to World Health Organization Standards. ${ }^{16}$

2. An estimated value $\geq 50 \mathrm{~mL}$ for the PVR using ultrasonography. ${ }^{17}$

During the same period, 256 cases of T2DM patients without DNB were randomly selected as the control group (T2DM group). The exclusion criteria of the two groups were:

1. People with type 1 diabetes mellitus.

2. People with benign prostatic hyperplasia (BPH).

3. People with a previous history of diseases, which are known to interfere with bladder or sphincter functions (eg, prostate or bladder cancer and previous genitourinary surgery).

4. Acute metabolic complications of diabetes containing ketoacidosis or hypertonic hyperglycemia.

5. Patients with serious dysfunctions of important organs such as the brain, the heart, and the lung amongst others.

\section{Methods and Calculations}

For each patient, informed consent was first obtained before collecting clinical data from personal medical records on patient admission. These data were specifically defined and included information such as anthropometric measurements (height and weight), demographic variables (sex and age), the duration of diabetes, any history of smoking and drinking, and comorbidity, as well as laboratory data (eg, bilirubin and hemoglobin). PLR and NLR were calculated as the ratio of platelets and neutrophils to lymphocytes, respectively, while the body mass index (BMI) was calculated as the weight divided by the height squared $(\mathrm{kg} / \mathrm{m} 2)$. Geriatric nutritional risk index (GNRI) was calculated from individually-obtained current body weight in $\mathrm{kg}$, the ideal body weight, the height in centimeters, and the serum albumin level. ${ }^{18}$ Finally, complications related to $\mathrm{T} 2 \mathrm{DM}$ were diagnosed according to criteria set out in specific guidelines.

\section{Statistical Analysis}

The Kolmogorov-Smirnov test was used to evaluate the distribution of the continuous variables. Normal distribution variables were expressed as means \pm standard deviations (SD) and were compared using the Student's $t$-test. On the other hand, variables exhibiting a non-normal 
distribution were expressed as median values and interquartile ranges before being compared using the MannWhitney $U$-test. In the case of counting data, values were expressed in frequencies and percentages (\%), with the two groups further compared using chi-square tests.
Finally, logistic regression was used to analyze the independent predictors of DNB. Taking DNB diagnosis as a positive result, the area under the receiver operating characteristic (ROC) curve along with the PLR cutoff point with the highest sensitivity and specificity were

Table I Baseline Patient Characteristics

\begin{tabular}{|c|c|c|c|}
\hline & DNB $(N=115)$ & T2DM $(\mathrm{N}=256)$ & P-value \\
\hline \multicolumn{4}{|l|}{ Sex } \\
\hline Male & $34(29.6 \%)$ & $174(68.0 \%)$ & $<0.001$ \\
\hline Female & $81(70.4 \%)$ & $82(32.0 \%)$ & \\
\hline Age & $63.3 \pm 13.0$ & $55.7 \pm 13.2$ & $<0.001$ \\
\hline BMI, kg/m2 & $22.2 \pm 3.78$ & $25.2 \pm 3.45$ & $<0.001$ \\
\hline $\mathrm{SBP}(\mathrm{mmHg})$ & $136 \pm 25.7$ & $136 \pm 18.6$ & 0.986 \\
\hline $\mathrm{DBP}(\mathrm{mmHg})$ & $76.8 \pm 14.2$ & $79.9 \pm 11.3$ & 0.024 \\
\hline Duration of diabetes (years) & $11.4 \pm 6.23$ & $6.68 \pm 5.90$ & $<0.001$ \\
\hline \multicolumn{4}{|l|}{ History (\%) } \\
\hline Smoking (current or ever) & 13 (II.3\%) & 92 (35.9\%) & $<0.001$ \\
\hline Drinking (current or ever) & 7 (6.1\%) & $66(25.8 \%)$ & $<0.001$ \\
\hline \multicolumn{4}{|l|}{ Comorbidity (\%) } \\
\hline Hypertension & $70(60.9 \%)$ & 115 (44.9\%) & 0.005 \\
\hline Diabetic nephropathy & $33(28.7 \%)$ & $5(2.0 \%)$ & $<0.001$ \\
\hline Atherosclerosis & 78 (67.8\%) & $126(49.2 \%)$ & $<0.001$ \\
\hline \multicolumn{4}{|l|}{ Laboratory data } \\
\hline $\mathrm{HbAlc} \%$ & $9.57 \pm 2.6 \mathrm{I}$ & $9.23 \pm 2.21$ & 0.503 \\
\hline FBG & $9.31 \pm 4.7 \mid$ & $7.91 \pm 3.65$ & $<0.001$ \\
\hline RBC & $3.78 \pm 0.637$ & $4.56 \pm 0.605$ & $<0.001$ \\
\hline WBC & $7.48 \pm 3.52$ & $7.08 \pm 1.89$ & 0.48 \\
\hline NLR & $4.04 \pm 3.32$ & $1.91 \pm 1.25$ & $<0.001$ \\
\hline PLR & $175 \pm 90 . \mid$ & $89.1 \pm 24.6$ & $<0.001$ \\
\hline $\mathrm{HB}$ & $110 \pm 23.0$ & $139 \pm 18.5$ & $<0.001$ \\
\hline Total bilirubin & $8.07 \pm 3.77$ & $|2.1 \pm 5.4|$ & $<0.001$ \\
\hline Direct bilirubin & $3.14 \pm 1.96$ & $4.48 \pm 2.70$ & $<0.001$ \\
\hline Indirect bilirubin & $4.93 \pm 2.30$ & $7.63 \pm 3.29$ & $<0.001$ \\
\hline Albumin & $34.5 \pm 6.64$ & $39.3 \pm 5.16$ & $<0.001$ \\
\hline ALT & $21.4 \pm 16.8$ & $30.2 \pm 22.8$ & $<0.001$ \\
\hline AST & $24.4 \pm 16.4$ & $27.1 \pm 15.6$ & 0.002 \\
\hline Blood urea nitrogen & $7.70 \pm 5.50$ & $5.59 \pm 2.15$ & $<0.001$ \\
\hline Creatinine & $85.2 \pm 62.7$ & $67.0 \pm 21.6$ & 0.097 \\
\hline Uric acid & $300 \pm 111$ & $333 \pm 96.6$ & $<0.001$ \\
\hline $\mathrm{TC}$ & $4.43 \pm 1.70$ & $4.71 \pm 1.19$ & 0.003 \\
\hline TG & $1.55 \pm 1.28$ & $2.21 \pm 1.89$ & $<0.001$ \\
\hline HDLc & $1.10 \pm 0.388$ & $0.99 \pm 0.236$ & 0.017 \\
\hline LDLc & $2.48 \pm 1.28$ & $2.59 \pm 0.844$ & 0.013 \\
\hline GNRI & $93.3 \pm 13.6$ & $140 \pm 33.7$ & $<0.001$ \\
\hline
\end{tabular}

Abbreviations: BMI, body mass index; SBP, systolic blood pressure; DBP, diastolic blood pressure; RBC, red blood cell; WBC, white blood cell; PLR, platelet-to-lymphocyte ratio; TB, total bilirubin; NLR, neutrophil-to-lymphocyte ratio; TG, triglycerides; TC, total cholesterol; GNRI, geriatric nutritional risk index; HB, hemoglobin; FBG, fasting blood glucose. 
obtained. All analyses were performed using IBM SPSS Statistics version 25.0. Differences were considered to be statistically significant for $\mathrm{p}$-values $<0.05$.

\section{Results}

\section{Comparison of General Data and Clinical Characteristics}

For this study, 371 patients were recruited, of which 115 had DNB (DNB group), and 256 had T2DM only (T2DM group). Table 1 summarizes the demographic characteristics and laboratory results of these two groups of patients. Compared to the T2DM group, values for the DNB group in terms of the percentage of women, diabetic nephropathy, atherosclerosis (AS), age, duration of diabetes, the levels of FBG, PLR, NLR and blood urea nitrogen (BUN) were significantly higher $(\mathrm{p}<0.05)$. On the other hand, smoking rates, drinking rates, the levels of DBP, BMI, RBC, hemoglobin (HB), mean platelet volume (MPV), total bilirubin (TB), direct bilirubin (DB), indirect bilirubin (IDB), uric acid (UA) and GNRI were all significantly lower than the values for the T2DM group $(\mathrm{p}<$ 0.05). Furthermore, compared with patients without DNB, the PLR and NLR were significantly higher for those with DNB $(\mathrm{p}<0.001)$.

Logistic regression analysis showed that the independent risk factors for DNB were duration of diabetes, PLR, TB, GNRI and HB (Table 2). In this case, forest plot was used to show the results more intuitively (Figure 1).

Table 2 Logistic Regression Analysis Showing Independent Predictors of DNB

\begin{tabular}{|l|c|c|c|}
\hline Variables & OR & 95\% Cl & p-value \\
\hline Sex & 0.65 & $0.285-1.485$ & 0.388 \\
Age & 0.983 & $0.946-1.02$ & 0.450 \\
Duration of diabetes & 1.127 & $1.059-1.203$ & 0.002 \\
Atherosclerosis & 0.963 & $0.413-2.227$ & 0.940 \\
BMI & 0.903 & $0.802-1.016$ & 0.155 \\
HbAIc & 1.074 & $0.905-1.284$ & 0.498 \\
HB & 0.96 & $0.936-0.983$ & 0.006 \\
PLR/IO & 1.408 & $1.248-1.617$ & $<0.001$ \\
NLR & 1.206 & $0.963-1.532$ & 0.184 \\
TB & 0.88 & $0.804-0.958$ & 0.016 \\
FBG & 1.051 & $0.953-1.159$ & 0.397 \\
UA & 0.998 & $0.994-1.002$ & 0.354 \\
GNRI & 0.953 & $0.927-0.972$ & $<0.001$ \\
\hline
\end{tabular}

Abbreviations: $\mathrm{BMI}$, body mass index; PLR, platelet-to-lymphocyte ratio; TB, total bilirubin; NLR, neutrophil-to-lymphocyte ratio; GNRI, geriatric nutritional risk index; HB, hemoglobin; UA, uric acid; FBG, fasting blood glucose; OR, odds ratio; $\mathrm{Cl}$, confidence interval.
Figure 2 shows that, as an independent risk factor for DNB, the cut-off value of PLR was 101.1949, while its sensitivity and specificity for the diagnosis of DNB were $89.6 \%$ and $23.4 \%$, respectively. Besides, the area under the curve was 0.899 (95\% CI: $0.865-0.932$ ).

\section{Discussion}

In the present study, we investigated the effectiveness of PLR/NLR in predicting the presence of diabetic neurogenic bladder. To the best of our knowledge, this is the first study demonstrating the relationships between DNB and PLR/NLR. Currently, research and sufficient understanding of DNB are still lacking, despite the fact that this condition is frequently diagnosed in patients with T2DM. In fact, it is known that bladder sensory impairment, increases in the post-void residual volume and decreases in detrusor contractility are typical urodynamic manifestations of the disease, ${ }^{19}$ but even though T2DM is a welldefined disease and relatively easy to diagnose, DNB tends to be latent with symptoms not appearing until the disease is in an advanced stage. ${ }^{20}$ Thus, it is important to find independent factors that are used for predicting the presence of DNB.

It is well known that metabolic abnormalities such as oxidative stress, glycolipid toxicity, and insulin resistance trigger-specific tissue inflammation. These, in turn, play an important role in the pathogenesis of T2DM, thereby suggesting that this disease is an autoinflammatory syndrome. ${ }^{21}$ The biology of DNB being multifactorial, its proposed pathogenesis includes ischemia, altered glucose metabolism, impaired axonal transport and superoxideinduced free-radical formation. ${ }^{22}$ Among these, hyperglycemia stimulation plays an important role as it results in increased oxidative phosphorylation in the bladder as well as in all insulin-independent tissues, thereby leading to significant oxidative stress. ${ }^{23}$ Oxidative stress is characterized by an imbalance between the antioxidant defenses of the body and the production of reactive oxygen species (free radicals). When an excessive number of free radicals react, they destroy major cellular components such as proteins, lipids and DNA. It is this oxidative stress that triggers an inflammatory response in many target tissues, with studies even reporting that this condition may potentiate inflammation in peripheral tissues along with the generation of more reactive oxygen species (ROS) in endothelial cells. ${ }^{24,25}$ These findings, therefore, suggest a correlation between the pathogenesis of DNB and inflammation. 


\section{Logistic forestplot}

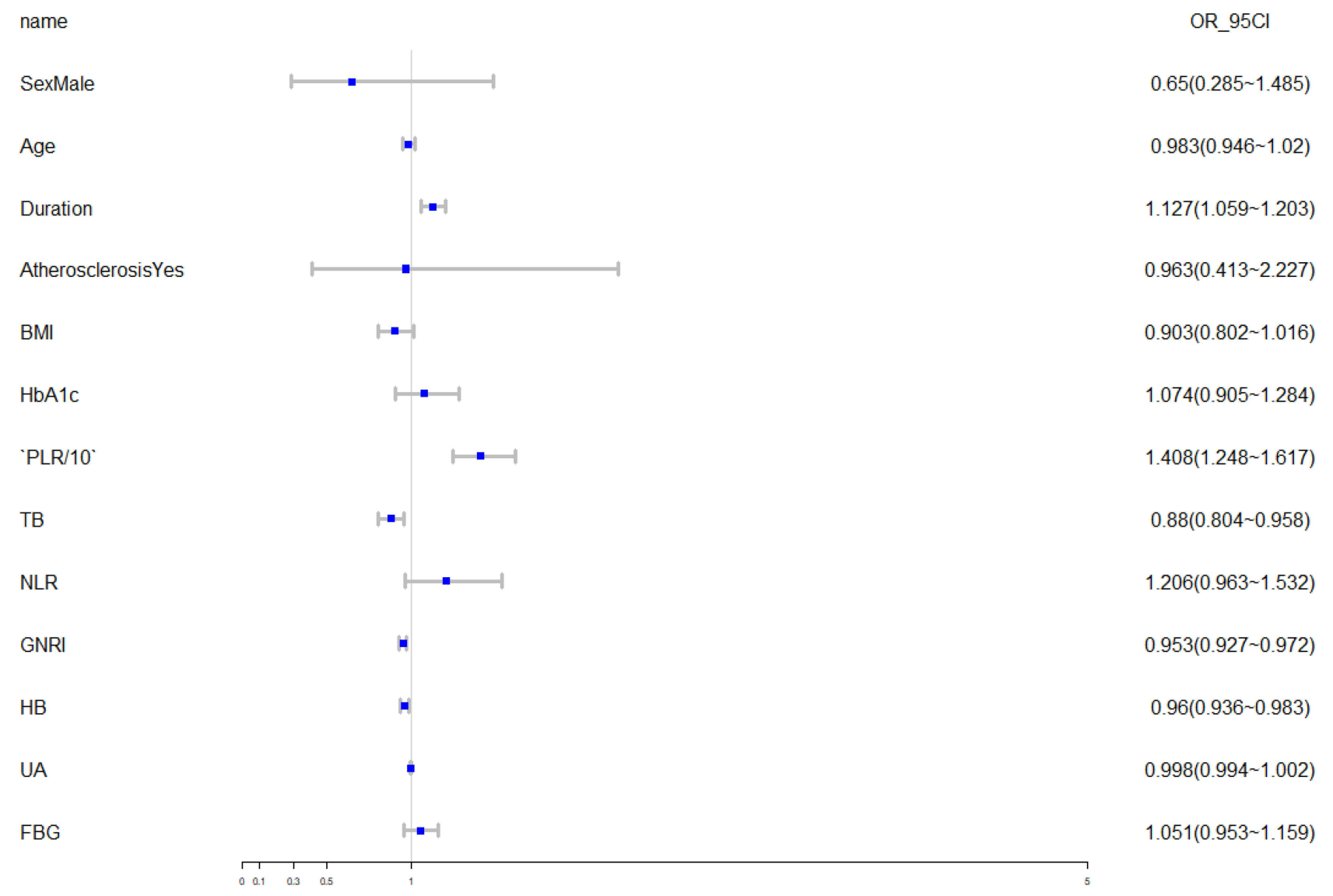

Figure I Logistic forest plot showing independent predictors of DNB.

Abbreviations: BMI, body mass index; PLR, platelet-to-lymphocyte ratio; TB, total bilirubin; NLR, neutrophil-to-lymphocyte ratio; GNRI, geriatric nutritional risk index; $\mathrm{HB}$, hemoglobin; UA, uric acid; FBG, fasting blood glucose; OR, odds ratio.

PLR and NLR are increasingly considered as indicative of systemic inflammations. ${ }^{26}$ Numerous studies have found PLR and NLR to have predictive significance, especially in the case of diabetes, various cancers, and for acute coronary syndromes. ${ }^{27}$ However, most studies on diabetes have, so far, only explored the relationships between PLR/NLR and microvascular complications. From the results of our study, it was found that, compared with diabetic patients without DNB, those with DNB had higher PLRs/NLRs and, hence, PLR and NLR were subsequently used as independent markers in predicting the presence of DNB. Through logistic regression analysis, PLR was found to be an independent risk factor in the presence of DNB, unlike NLR for which no independent association with DNB was found. Therefore, there is no ROC analysis for NLR to define its predictive value.

Increasing evidence has shown that PLR is associated with diabetes and its complications, with the predictive value of this marker having already been proven in numerous studies. For instance, according to Hudzik et al, PLR was considered an independent risk factor in diabetic patients for early and late mortality. ${ }^{28}$ Similarly, Yue et al found that PLR was significantly increased during the onset of diabetic retinopathy (DR) ${ }^{28}$ while Zhang et al reported that the PLR for patients with diabetic foot ulcers (DFU) increased significantly and was positively correlated with Wagner DFU grade. ${ }^{29}$

As a potential biomarker reflecting inflammation responses, many studies have reported that PLR is positively correlated with traditional inflammatory markers. Since PLR is calculated as the ratio of platelets to lymphocytes, a high PLR can either occur as a result of the platelet count being high or the lymphocyte count being low. In previous studies, elevated platelet counts have been correlated with proinflammatory substances and acute-phase reactants, such as tumor necrosis factor a, high-sensitivity C-reactive protein (hsCRP), 


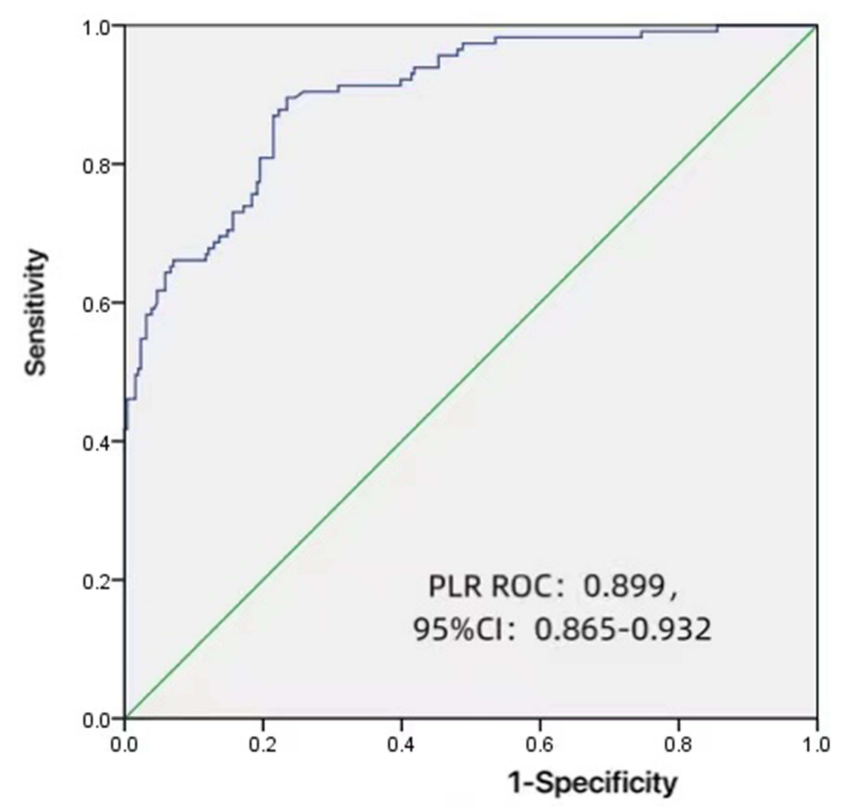

Figure 2 Receiver-operating characteristics (ROC) curve analysis for platelet-tolymphocyte ratio as a predictor of the severity of diabetic neurogenic bladder. Abbreviations: ROC, receiver operating characteristic; $\mathrm{Cl}$, confidence interval; PLR, platelet-to-lymphocyte ratio.

interleukin-1, and interleukin- $6 .{ }^{30}$ In fact, high platelet counts may also reflect an increased release of inflammatory mediators as well as increased thrombocyte activation, resulting in destructive inflammatory response and thrombotic states. ${ }^{31}$ Similarly, the second component of PLR, the lymphocyte count, plays an important role in inflammatory response. Lymphocytes regulate inflammatory responses and accelerated apoptosis of lymphocytes can lead to lymphopenia. ${ }^{32}$ Possible etiologies of DNB, such as hyperglycemic stimulation that occurs through oxidative stress and inflammatory responses, can impair the bladder endothelial cell and axonal transport, hence leading to abnormal bladder dysfunction. These explain why PLR can have predictive value regarding the onset of DNB.

Our study still has several limitations. First, it was undertaken in a single center while involving a relatively small sample size. Thus, the results may not be representative of all patients with diabetic neurogenic bladder. Furthermore, being a case-control study design, it could not be investigated whether controlling the PLR could affect DNB outcome. Finally, due to the large age span of patients with diabetic neurogenic bladder and the time of onset is uncertain, the basic characteristics of the research objects were not well balanced.

\section{Conclusions}

In conclusion, our study found that PLR and NLR values were significantly higher in patients with DNB. PLR was a reliable predictive biomarker for DNB diagnosis, but considering the severe complications associated with this condition, it is recommended to use PLR mainly during the early stages to predict the presence of DNB. Periodic residual urine or urodynamic measurement and early intervention are important measures in patients with elevated PLR. Furthermore, patients with elevated PLR values should be more seriously cared for in clinical settings.

\section{Ethics Statement}

This study was conducted in accordance with the Declaration of Helsinki, and it was approved by the ethics committee in Clinical Research of the First Affiliated Hospital of Wenzhou Medical University; Acceptance Number: KY2021-R089.

\section{Acknowledgments}

The authors thank the staff at the Department of Endocrinology and Metabolism, the First Affiliated Hospital of Wenzhou Medical University, and all the patients who participated in the study. Yiying Liu and Xin Wang are co-first authors of this study.

\section{Disclosure}

The authors report no conflicts of interest in this work.

\section{References}

1. Wild S, Roglic G, Green A, Sicree R, King H. Global prevalence of diabetes: estimates for the year 2000 and projections for 2030 . Diabetes Care. 2004;27(5):1047-1053. doi:10.2337/diacare.27.5. 1047

2. Yuan Z, Tang Z, He C, Tang W. Diabetic cystopathy: a review. J Diabetes. 2015;7(4):442-447. doi:10.1111/1753-0407.12272

3. Peng X, Li S, An Z, Li L. [Application value of American urological association symptom index score in female patients with type 2 diabetic neurogenic bladder]. Sichuan Da Xие Xиe Bao Yi Xue Ban. 2019;50(4):566-570. Chinese

4. Kebapci N, Yenilmez A, Efe B, Entok E, Demirustu C. Bladder dysfunction in type 2 diabetic patients. Neurourol Urodyn. 2007;26 (6):814-819. doi:10.1002/nau.20422

5. Shiny A, Bibin YS, Shanthirani CS, et al. Association of neutrophil-lymphocyte ratio with glucose intolerance: an indicator of systemic inflammation in patients with type 2 diabetes. Diabetes Technol Ther. 2014;16(8):524-530. doi:10.1089/dia.2013.0264

6. Horne B, Anderson J, John J, et al. Which white blood cell subtypes predict increased cardiovascular risk? J Am Coll Cardiol. 2005;45 (10):1638-1643. doi:10.1016/j.jacc.2005.02.054

7. Esser N, Legrand-Poels S, Piette J, Scheen AJ, Paquot N. Inflammation as a link between obesity, metabolic syndrome and type 2 diabetes. Diabetes Res Clin Pract. 2014;105(2):141-150. doi:10.1016/j.diabres.2014.04.006

8. Vozarova B, Weyer C, Lindsay R, Pratley R, Bogardus C, Tataranni P. High white blood cell count is associated with a worsening of insulin sensitivity and predicts the development of type 2 diabetes. Diabetes. 2002;51(2):455-461. doi:10.2337/diabetes.51.2.455 
9. Azab B, Shah N, Akerman M, McGinn J. Value of platelet/lymphocyte ratio as a predictor of all-cause mortality after non-ST-elevation myocardial infarction. J Thromb Thrombolysis. 2012;34(3):326-334. doi:10.1007/s11239-012-0718-6

10. Liu J, Du J, Fan J, et al. The neutrophil-to-lymphocyte ratio correlates with age in patients with papillary thyroid carcinoma. ORL J Otorhinolaryngol Relat Spec. 2015;77(2):109-116. doi:10.1159/ 000375534

11. Semeniuk-Wojtaś A, Lubas A, Stec R, Syryło T, Niemczyk S, Szczylik C. Neutrophil-to-lymphocyte ratio, platelet-to-lymphocyte ratio, and $\mathrm{C}$-reactive protein as new and simple prognostic factors in patients with metastatic renal cell cancer treated with tyrosine kinase inhibitors: a systemic review and meta-analysis. Clin Genitourin Cancer. 2018;16(3):e685-e693. doi:10.1016/j. clgc.2018.01.010

12. Akbas E, Demirtas L, Ozcicek A, et al. Association of epicardial adipose tissue, neutrophil-to-lymphocyte ratio and platelet-tolymphocyte ratio with diabetic nephropathy. Int J Clin Exp Med. 2014;7(7):1794-1801.

13. Mineoka Y, Ishii M, Hashimoto Y, Yamashita A, Nakamura N, Fukui M. Platelet to lymphocyte ratio correlates with diabetic foot risk and foot ulcer in patients with type 2 diabetes. Endocr J. 2019;66 (10):905-913. doi:10.1507/endocrj.EJ18-0477

14. Wang J, Chen Z, Yang K, et al. Association between neutrophil-tolymphocyte ratio, platelet-to-lymphocyte ratio, and diabetic retinopathy among diabetic patients without a related family history. Diabetol Metab Syndr. 2020;12:55. doi:10.1186/s13098-020-00562-y

15. Wang C, Kuo H. Urothelial dysfunction and chronic inflammation in diabetic patients with overactive bladder. Low Urin Tract Symptoms. 2017;9(3):151-156. doi:10.1111/luts.12126

16. Alberti K, Zimmet P. Definition, diagnosis and classification of diabetes mellitus and its complications. Part 1: diagnosis and classification of diabetes mellitus provisional report of a WHO consultation. Diabet Med. 1998;15(7):539-553. doi:10.1002/(SICI) 1096-9136(199807)15:7<539::AID-DIA668>3.0.CO;2-S

17. Sakakibara R, Takahashi O, Nishimura $H$, et al. The relationship between bladder, periarterial and somatic neuropathy in diabetes. Intern Med Adv Publ. 2018;57(15):2165-2168. doi:10.2169/internalmedicine.9749-17

18. Bouillanne O, Morineau G, Dupont C, et al. Geriatric nutritional risk index: a new index for evaluating at-risk elderly medical patients. $\mathrm{Am}$ J Clin Nutr. 2005;82(4):777-783. doi:10.1093/ajen/82.4.777

19. Kaplan S, Blaivas J. Diabetic cystopathy. J Diabet Complications. 1988;2(3):133-139. doi:10.1016/S0891-6632(88)80024-2

20. Frimodt-Møller C. Diabetic cystopathy: epidemiology and related disorders. Ann Intern Med. 1980;92:318-321. doi:10.7326/00034819-92-2-318
21. Akash MSH, Rehman K, Chen S. Role of inflammatory mechanisms in pathogenesis of type 2 diabetes mellitus. J Cell Biochem. 2013;114 (3):525-531. doi:10.1002/jcb.24402

22. Apfel S. Neurotrophic factors and diabetic peripheral neuropathy. Eur Neurol. 1999;41(Suppl. 1):27-34. doi:10.1159/000052077

23. Inouye B, Hughes F, Jin H, et al. Diabetic bladder dysfunction is associated with bladder inflammation triggered through hyperglycemia, not polyuria. Res Rep Urol. 2018;10:219-225. doi:10.2147/ RRU.S177633

24. Akash M, Shen Q, Rehman K, Chen S. Interleukin-1 receptor antagonist: a new therapy for type 2 diabetes mellitus. J Pharm Sci. 2012;101(5):1647-1658. doi:10.1002/jps.23057

25. Hayden M, Sowers J. Isletopathy in type 2 diabetes mellitus: implications of islet RAS, islet fibrosis, islet amyloid, remodeling, and oxidative stress. Antioxid Redox Signal. 2007;9(7):891-910. doi:10.1089/ars.2007.1610

26. Yang W, Wang X, Zhang W, et al. Neutrophil-lymphocyte ratio and platelet-lymphocyte ratio are 2 new inflammatory markers associated with pulmonary involvement and disease activity in patients with dermatomyositis. Clin Chim Acta. 2017;465:11-16. doi:10.1016/j. cca.2016.12.007

27. Yue S, Zhang J, Wu J, Teng W, Liu L, Chen L. Use of the monocyte-to-lymphocyte ratio to predict diabetic retinopathy. Int J Environ Res Public Health. 2015;12(8):10009-10019. doi:10.3390/ijerph120810009

28. Hudzik B, Szkodzinski J, Gorol J, et al. Platelet-to-lymphocyte ratio is a marker of poor prognosis in patients with diabetes mellitus and ST-elevation myocardial infarction. Biomark Med. 2015;9 (3):199-207. doi:10.2217/bmm.14.100

29. Zhang K, Ding S, Lyu X, Tan Q, Wang Z. Correlation between the platelet-to-lymphocyte ratio and diabetic foot ulcer in patients with type 2 diabetes mellitus. J Clin Lab Anal. 2021;35(4):e23719. doi:10.1002/jcla.23719

30. Alexandrakis M, Passam F, Moschandrea I, et al. Levels of serum cytokines and acute phase proteins in patients with essential and cancer-related thrombocytosis. Am J Clin Oncol. 2003;26 (2):135-140. doi:10.1097/01.COC.0000017093.79897.DE

31. Ozcan Cetin E, Cetin M, Aras D, et al. Platelet to lymphocyte ratio as a prognostic marker of in-hospital and long-term major adverse cardiovascular events in ST-segment elevation myocardial infarction. Angiology. 2016;67(4):336-345. doi:10.1177/ 0003319715591751

32. Chen $\mathrm{W}, \mathrm{Chen} \mathrm{K}, \mathrm{Xu} \mathrm{Z}$, et al. Neutrophil-to-lymphocyte ratio and platelet-to-lymphocyte ratio predict mortality in patients with diabetic foot ulcers undergoing amputations. Diabetes Metab Syndr Obes. 2021;14:821-829. doi:10.2147/DMSO.S284583

Diabetes, Metabolic Syndrome and Obesity: Targets and Therapy

Dovepress

Publish your work in this journal

Diabetes, Metabolic Syndrome and Obesity: Targets and Therapy is an international, peer-reviewed open-access journal committed to the rapid publication of the latest laboratory and clinical findings in the fields of diabetes, metabolic syndrome and obesity research. Original research, review, case reports, hypothesis formation, expert opinion and commentaries are all considered for publication. The manuscript management system is completely online and includes a very quick and fair peer-review system, which is all easy to use. Visit http://www.dovepress.com/testimonials.php to read real quotes from published authors. 